\title{
Animal Models of COVID-19 for Vaccine and Therapeutic Development
}

\author{
Maggie $\mathrm{Hu}$ \\ Urbana High School, Ijamsville, MD, 21754, USA ; maggieq2003@gmail.com
}

\begin{abstract}
The emergence of severe acute respiratory syndrome coronavirus 2 (SARS-CoV-2) in late 2019 poses an imminent global health crisis. There is an urgent need to develop effective therapeutics and vaccines for the treatment and prevention of COVID-19. Animal models are a critical avenue of study to identify key molecular mechanisms and establish the safety and efficacy of interventions. SARS-CoV-2 infection of mice, guinea pigs, ferrets, nonhuman primates, and other laboratory animals has been established as models of COVID-19. They contribute significantly to the discovery and in vivo testing of vaccine and therapeutic candidates. In this study, I present a comprehensive literature review on the different animal models available to assess SARS-CoV-2 infection. The standards of a faithful animal model are reviewed to evaluate the strengths and limitations of each species in reproducing aspects of human disease. Differences in anatomy and physiopathology among the animal models present difficulties in representing the complexity and complete spectrum of human disease. However, nonhuman primates have a closer phylogenetic relation with humans compared to other animals, making them models of particular utility. Their applications for vaccine and therapeutic development are discussed to highlight their critical role in present and future research efforts against the pandemic.

KEYWORDS: Translational Medical Sciences; Pre-Clincal Studies; COVID-19; Nonhuman Primate Model; Vaccine Therapeutic Development.
\end{abstract}

\section{- Introduction}

Severe acute respiratory syndrome coronavirus 2 (SARS$\mathrm{CoV}-2)$ is the cause of the novel coronavirus disease 2019 (COVID-19). On 30 January 2020, the World Health Organization (WHO) declared the pandemic a global emergency of public health. ${ }^{1}$ At the time of writing, infections are still rapidly spreading, increasing the number of reported daily cases. With no approved vaccine, drug, or treatment for COVID-19, a dire medical need continues to exist. Clinical trials are currently underway for prevention and interventions of the disease, ${ }^{2}$ but it is still paramount to continue fundamental research on the virus for a better understanding of the pathogenesis and development strategies of therapeutic agents.

Clinical testing is typically a top predictor of drug and therapeutic efficacy, but it must proceed with caution due to possible unintended side effects, such as disease enhancement. ${ }^{3}$ For this, animal models that can successfully reproduce the behavior and the pathology of COVID-19 are an important tool of study. These models, ranging from laboratory mice to monkeys, provide insight on the usefulness and effects of vaccines and antiviral therapeutic agents. Although no existing model of SARS-CoV-2 infection completely reproduces every feature of severe COVID-19 in humans, nonhuman primates share many aspects of their anatomy and physiology with humans, making them prime candidates of study.

Currently, nonhuman primate models are being used to investigate the pathogenesis of SARS-CoV-2 infection and for preclinical evaluation of drugs and vaccines against the virus.
All animal models present considerable differences in disease susceptibility and transmission, and once infected, they show varying degrees of replication. The variances in disease progression among the models overlaps with human infection. Each model provides insights into the disease mechanism, and notably, nonhuman primates resemble human systems more closely than other phylogenetically distant models. As such, they have been commonly used to evaluate diseases. This review will focus primarily on the nonhuman primate models of disease, including studies of rhesus macaques (Macaca mulatta), cynomolgus macaques (Macaca fascicularis), and more. Their use as a model of human COVID-19 for SARS-CoV-2 therapeutic development will be addressed to show how they have contributed and will continue to aid global efforts against this disease.

\section{Different Animal Models for COVID-19}

Requirements of animal models for COVID-19:

A faithful animal model for SARS-CoV-2 infection should reflect the clinical signs, viral replication, and pathology seen in humans. It would ideally exhibit severe pneumonia, multiple organ failure, fatality, or other severe COVID-19 symptoms. ${ }^{4}$ The models do not need to reproduce all aspects of the disease to aid in human treatment efforts. Still, researchers around the world continue to develop models of the disease with various species to gather the most accurate results. They have used many models, especially those that were historically used for other coronaviruses, to best represent the novel coronavirus. These animal models permit an understanding of the disease and its complex pathophysiology through systemic virus host 
interactions studied within the models. Since the pathology of the disease is reproducible in a range of animal models, numerous different animals and species have been useful to study disease and test candidate therapeutic compounds.

Among the many different animals that have been studied, such as laboratory mice, ferrets, cats, hamsters, nonhuman primates, and more, ${ }^{5}$ there are advantages and disadvantages that affect experimental results. These animals have been used as models for other respiratory viruses before, such as SARS-CoV-1 and other previous and existing diseases. ${ }^{4}$ Scientists often test the susceptibility of various animals to SARS-CoV-2 to model pathogenic mechanisms and control interventions, ${ }^{6}$ which is important for preliminary studies. Studies have found this virus replicates poorly in dogs, pigs, chickens, and ducks, but many other small and large animals have served as useful tools of study. ${ }^{5}$

\section{Small animal models:}

SARS-CoV-2 infection has been studied in many small animal models that often reproduce faster and are easy to handle, making them advantageous for urgent studies. The use of mice and hamsters has proven to be cost-effective and efficient. It was found that in wild mice, the novel coronavirus cannot invade cells through mouse orthologue of the human SARS-CoV-2 receptor, ACE2. Rodent studies were initially a limited avenue of investigation since no mice or rodents AFAIK could be infected, but through the use of human ACE2 transgenic mice, researchers were able to successfully overcome the natural resistance of mice to the infection. ${ }^{7}$ Results with the use of these subjects showed human signs of the disease, such as weight loss, virus replication in the lungs, and interstitial pneumonia. In the model developed by Dinnon et al., ${ }^{8}$ they performed this with two amino acid substitutions in the viral SPIKE protein based on molecular modeling and rodent ACE2. Despite these alterations, murine models were limited in their evaluation of COVID-19 pathogenesis due to their more acute differences in their genetics, anatomy, and physiology. Golden et al. developed a human ACE2 transgenic mice mode and used it to evaluate the pathogenesis of SARS-CoV-2 in male and female mice expressing the human ACE2 under the control of the keratin 18 promoter (K18). ${ }^{9}$ In contrast to nontransgenic mice, intranasal exposure of K18-hACE2 animals to 2 different doses of SARS-CoV-2 resulted in acute disease, including weight loss, lung injury, brain infection, and lethality. Smith et al. evaluated the immunogenicity of a DNA vaccine INO-4800 in mice and guinea pigs. ${ }^{10}$ Following immunization of mice and guinea pigs with INO-4800 they measured antigen-specific $\mathrm{T}$ cell responses, functional antibodies that neutralize the SARS-CoV-2 infection with humoral immune response.

In golden hamsters, researchers have found they could be consistently infected by SARS-CoV-2, clinically resembling upper and lower respiratory tract infection manifestations in humans. The animals showed increasing respiratory rate, decreasing activity, and progressive weight loss. These symptoms were all similar to infected humans. ${ }^{11}$ Since the hamster ACE2 is characterized by a higher degree of homology to humans ACE2 compared to mice, researchers discovered dis- tinct findings, such as the possibility of convalescent plasma decreasing viral burden. The hamster model was naturally susceptible to infection. Golden hamsters are genetically dissimilar from humans in important aspects, which slightly diminishes their value as suitable models of study. ${ }^{6}$ Overall, small animals like rodents lack many key human features that other models, such as primates, carry. Examples of these include sophisticated brain structure, developed immune systems, motor skills, metabolic functions, and more. ${ }^{12}$ Thus, for further reproduction of pathology, large animal models may often be preferable.

\section{Large animal models:}

Large animal models often include ferrets and cats. Ferrets have successfully modeled respiratory disease and its transmission in the past, such as cases of influenza and SARS-CoV-1. For SARS-CoV-2, they have the advantage of a cough reflex-like humans that rodents do not have. ${ }^{13}$ Since SARS-CoV-2 replicates efficiently in the upper respiratory tract, results from ferrets are useful to evaluate. Although fatality was not often observed in infected ferrets, the animals showed higher body temperature and virus replication. Additionally, they experienced viral shedding and acute bronchiolitis in the lungs. ${ }^{5}$ The findings suggest the ferret model will be especially helpful for studies of cough and fever symptoms. Cats, although not a traditional model, have also been studied, which primarily followed from reports showing cases of domestic animals infected. ${ }^{14}$ These animals exhibited some signs of disease, such as pulmonary oedema. Notably, it was discovered that SARS-CoV-2 in cats was transmissible via an airborne route. In comparison to ferrets, the cat model has a more limited transmission of disease, and tested subjects were noted to be particularly aggressive. Shi et al. experienced difficulty collecting samples and data from subadult cats, as the subjects responded violently to their attempts and prevented the researchers from obtaining samples live. This hardship presents additional limitations to cats for study, as well as the ethical concerns in using a domesticated species in close proximity to humans. Ultimately, the high susceptibility of cats to infection has been warned as a consideration for human elimination of COVID-19.

As shown in Figure 1, many animals have been used to study SARS-CoV-2 infection to gain a clearer understanding of the disease before clincal trials proceed. Although animals such as ferrets and cats showed promising findings for specific areas of study, they presented limitations that could be explored with use of other animal models. For example, studies have found that inoculated ferrets did not exhibit lower respiratory pathological findings, and there was no oedema or pulmonary replication caused by the infection. ${ }^{13}$ Cats are not typically used for research for humanitarian and safety reasons. A more often used model among the many large animal subjects (Figure 1) are nonhuman primates.

\section{Nonbuman Primate Models for COVID-19:}

Historically, nonhuman primates have been the main animals that allow for efficient response and research into emerging viruses. These characteristics are of utmost importance during the current pandemic. ${ }^{12}$ Macaques and their 
similarities to humans in almost all anatomical traits have made them a particularly accurate model for understanding the pathogenesis of SARS-CoV-2 infection. They respond similarly to many human immulogic, pathological agents, developing signs, and host responses humans experience. As one of the most readily available models, they are indispensable to medical research and development. ${ }^{15}$ The macaques appear naturally susceptible to SARS-CoV-2 infection and display many features of human COVID-19 disease, but it ranges mainly from mild to asymptomatic infection. Studies show that macaques and humans share extensive clinical manifestations of SARS-CoV-2, including pulmonary signs and symptoms. ${ }^{16}$ Researchers can monitor the disease progression of SARS-COV-2 infection in macaques by measuring similar parameters as tested in humans, from radiographic imaging and body fluid samples.

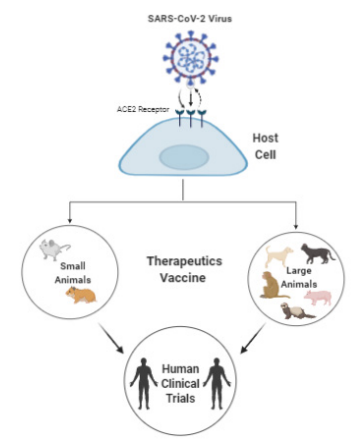

Figure 1: SARS-CoV-2 infection and animal models for vaccine and therapeutic development for the treatment of COVID-19. Small animals such as mice and golden hamsters are pictured on the right. Large animal candidates include dogs, cats, pigs, ferrets, and notably, nonhuman primates. These models are typically studied before clinical testing. The figure was created using BioRender (wwww.biorender.com).

\section{Established macaque models:}

Macaques are an animal model of particular utility because they replicate many features of human disease. Most studies to date have used the Old World Monkey rhesus macaque model, but other non-human primate species, such as Old World cynomolgus macaques, African green monkeys, and New World common marmosets have also been studied. The animals are inoculated via different routes, through intratracheal, intranasal, ocular, and/or oral routes. Clinical observations are made days after to varying degrees: for example, weight loss is sometimes observed moderately, sometimes in all of the animals or rarely. ${ }^{15,16}$ Many develop the respiratory disease and develop some pulmonary pathology - radiographs often show pulmonary infiltrates, the hallmark of human infection, and their viral shedding pattern is similar to humans. Typically, the rhesus macaque models mild disease observed in the majority of human cases. ${ }^{17}$ Fewer studies have been performed using cynomolgus macaques, some of which did not show weight loss or transient respiratory disease like other studies with rhesus macaques did. ${ }^{16}$ This suggests the cynomolgus macaque species models less severe cases of the infection. emerging viruses. These characteristics are of utmost importance during the current pandemic. ${ }^{12}$ Macaques and their similarities to humans in almost all anatomical traits have made them a particularly accurate model for understanding the pathogenesis of SARS-CoV-2 infection.

Due to differences in strain, inoculation route, age, and other factors, $\mathrm{Lu}$ et al. conducted a study to standardize results between cynomolgus macaques, rhesus macaques, and common marmosets. They found that out of the three, rhesus macaques were the most suitable and susceptible model to SARS-CoV-2. ${ }^{18}$ More recently, the African green monkey model has been established as a reliable model, showing high levels of virus replication and development of the more pronounced respiratory disease than the rhesus macaque and cynomolgus macaque species. They needed a much lower dose of SARS-CoV-2 to cause this response in comparison to other nonhuman primate studies, suggesting their use is more cost-effective as well. ${ }^{19}$ African green monkeys should be further evaluated for these advantageous findings alongside other species studies.

\section{Aged Macaque Model:}

Individuals of the aged population have been found to be at a greater risk of developing severe progression of COVID-19. The macaque models are essential for understanding the aging process and identifying interventions of disease in the aged, as they age similarly to humans. Yu et al. conducted a study with five rhesus macaques of different ages, 3 old (15 years) and 2 young (3-5 years), by inoculating them with SARS-CoV-2 infection. ${ }^{20}$ The monkeys showed clinical signs but no significant change in body temperature and other typical signs of human infection. ${ }^{21}$ Overall, the older monkeys infected with SARS-CoV-2 experienced more severe interstitial pneumonia and more viral load than young monkeys demonstrated through more viral replication, chest $\mathrm{X}$-rays, histopathological changes and immune responses. The availability of older primate poses a timely limitation on researchers because many of the test candidates would need to wait years to age. Still, this aged animal model reinforced increased risks for aged populations and serves as an important investigative path for modeling severe cases.

\section{Reinfection Macaque Model:}

Bao et al. developed a short-term infection rechallenge macaque model to study whether patients have a risk of reinfection and whether seroconversion provides immunity against SARS-CoV-2 in nonhuman primate rhesus macaque models. ${ }^{7}$ In this report, the model was characterized by interstitial pneumonia and systemic viral dissemination in the respiratory and gastrointestinal tracts, as well as similar characteristics of pathogenicity found in other studies. ${ }^{15,16,18}$ The monkeys were protected against short term reinfection, showing a lack of viral shedding, histopathological changes, and other signs that suggest protective immunity. Other studies found similar results, indicating that the neutralizing antibody might provide protection from the secondary infection but COVID-19 patients have tested positive twice. ${ }^{22}$ Thus, more studies with a longer interval between the primary challenge and rechallenge are necessary to further understand the host-virus interactions and protective mechanism against SARS-CoV-2. Overall, this susceptibility of macaques and promising display of a similar disease spectrum in the models 
has allowed researchers to move forward with preclinical testing of vaccines and drugs.

\section{Animal Models for Vaccine and Therapeutic Development Vaccine:}

Applications of the macaque model in vaccine and therapeutics development for the treatment of COVID-19 are summarized in Table 1 . The ChAdOx MERS vaccine has been tested in mice and rhesus macaques infected with MERS-CoV. ${ }^{23}$ In this study, animals were inoculated with a dose of virus that was higher than the amount most humans are exposed to. The results showed broad protection and immune responses in the animals that allowed them to adapt to the single dose to SARS-CoV-2 infection. Researchers tested vaccine efficacy on rhesus macaques once again, observing that it effectively prevented lung damage, caused no signs of virus replication in the lungs, and significantly lowered levels of respiratory disease. There was no sign of immune enhanced disease after vaccination, which had been a common problem for previous coronaviruses. These promising results pave a way for further development, as $\mathrm{ChAdOx} 1$ vaccines can be produced rapidly. In fact, clinical trials are currently underway with human volunteers. This is part of the ongoing phase I clinical trials, where safety, immunogenicity, and efficacy against symptomatic PCR-positive COVID-19 disease are assessed in randomized human subjects.

$\mathrm{Yu}$ et al. confirmed the therapeutic effect of another potential DNA vaccine candidate by testing the efficacy of a set of prototype DNA vaccines expressing various forms of the SARS-CoV-2 spike (S) protein. ${ }^{17}$ They assessed their immunogenicity and protective efficacy against SARS-CoV-2 viral challenge in rhesus macaques. After being vaccinated, animals developed humoral and cellular immune responses, including neutralizing antibody titers at levels comparable to those found in convalescent humans and macaques infected with SARS-CoV-2. This showed that animals had vaccine protection against SARS-CoV-2 in nonhuman primates. The researchers defined NAb titers as an immune correlate of protection, which will be useful for vaccine development considerations. Although data from this study was restricted to DNA vaccines, findings may apply to other gene-based vaccines as well, including RNA vaccines and recombinant vector-based vaccines. If this NAb correlate is further shown across multiple vaccine studies in nonhuman primates and humans, it would be a simple and useful benchmark for clinical development of SARS-CoV-2 vaccines.

In another preclinical study, Gao et al. tested a purified inactivated vaccine candidate, $\mathrm{PiCoVacc}$, by immunizing rhesus macaques. ${ }^{24}$ The animals showed no clinical abnormalities after the vaccine when compared to the unvaccinated control group. Researchers then inoculated the animals with SARSCOV-2, and the vaccinated macaques were protected against infection, showing mild and focal histopathological changes in the lungs. Their data verified the protective efficacy: histopathological evaluations of various organs demonstrated that PiCoVacc did not cause any notable pathology in macaques. With no observation of infection enhancement or immunopathological exacerbation as well as complete protection with a higher dose of $\mathrm{PiCoVacc}$, this vaccine shows a promising outlook for future human use and clinical development of a vaccine.

\section{Antiviral drugs:}

Remdesivir has been proven effective against MERS-CoV and SARS-CoV-1 infection in the past. ${ }^{25}$ Williamson et al. tested remdesivir using the established rhesus macaque model of SARS-CoV-2 infection that resulted in lower respiratory tract disease. ${ }^{26}$ In contrast to vehicle-treated animals, remdesivir treated rhesus macaques did not show signs of respiratory disease and had reduced pulmonary infiltrates on radiographs. Viral loads of remdesivir treated animals were significantly lower and there was a clear reduction in damage to the lung tissue. Thus, they showed that therapeutic remdesivir treatment initiated early during infection has a clear clinical benefit in the rhesus macaques. This supported early remdesivir treatment in COVID-19 patients to prevent severe pneumonia progression.

Hydroxychloroquine (HCQ) has also been extensively investigated in various animal models, but the results have not been as promising. Studies have shown no significant prophylactic or therapeutic benefit of HCQ following in vivo infection in animal disease models, like nonhuman primates. ${ }^{27}$ When tested in the rhesus macaque model, both high doses and the standard human dose yielded no significant benefit, clinical outcome, or reduction in virus replication or shedding in the upper and lower respiratory tract. In another study, Maisonnasse et al. infected cynomolgus macaques instead and administered HCQ daily to assess the anti-viral efficacy. No antiviral activity in respiratory compartments nor clinical efficacy of treatment was determined, regardless of the timing of treatment initiation. ${ }^{28}$ Not only do these results exhibit the common discrepancy between results from in vitro classic assays and in vivo experiments, but they also fail to support the use of HCQ in prophylaxis or treatment of COVID-19.

\section{- Discussion}

Table 1.

\begin{tabular}{|c|c|c|c|c|}
\hline $\begin{array}{l}\text { Animal } \\
\text { Model }\end{array}$ & $\begin{array}{l}\text { Therapeutic } \\
\mathbf{s}\end{array}$ & & Outcome & Ref \\
\hline Mouse & DNA Vaccine & INO-4800 & neutralizing antibody response & 10 \\
\hline Mouse & DNA Vaccine & PiCoVacc & protective immune response & 23 \\
\hline Guinea Pig & DNA Vaccine & INO-4800 & humoral immune response & 10 \\
\hline $\begin{array}{l}\text { Macaques } \\
\text { (rhesus) }\end{array}$ & DNA Vaccine & NAb & humoral immune responses & 17 \\
\hline $\begin{array}{l}\text { Macaques } \\
\text { (rhesus) }\end{array}$ & Vaccine & PiCoVacc & $\begin{array}{l}\text { mild and focal histopathological changes } \\
\text { in the lungs }\end{array}$ & 23 \\
\hline $\begin{array}{l}\text { Macaques } \\
\text { (rhesus) }\end{array}$ & DNA Vaccine & $\begin{array}{l}\text { ChAdOx1 } \\
\text { MERS }\end{array}$ & humoral immune responses & 22 \\
\hline $\begin{array}{l}\text { Macaques } \\
\text { (rhesus) }\end{array}$ & Antivirals & Remdesivir & no reduction in virus shedding observed & 25 \\
\hline $\begin{array}{l}\text { Macaques } \\
\text { (rhesus) }\end{array}$ & Antivirals & $\mathrm{HCQ}$ & $\begin{array}{l}\text { no significant benefit clinical outcome or } \\
\text { reduction in virus replication or shedding } \\
\text { in the upper and lower respiratory tract }\end{array}$ & 26 \\
\hline $\begin{array}{l}\text { Macaques } \\
\text { (cynomolgu } \\
\text { s) }\end{array}$ & Antivirals & $\mathrm{HCQ}$ & $\begin{array}{l}\text { no antiviral activity in respiratory } \\
\text { compartments and clinical efficacy of } \\
\text { treatment determined }\end{array}$ & 27 \\
\hline
\end{tabular}

Even with their close mimicry of the human disease spectrum, macaques are not a perfect proxy for COVID-19. Nonhuman primates are extremely expensive to care for and house in comparison to other models, even more so than hu- 
mans. Studies are also frequently limited in the number of animals in their studies, partly due to high maintenance costs and space requirements. Furthermore, equipment to conduct studies is expensive and handling the primates is often labor-intensive. Their similar aging and development periods make them useful models for age-related studies, but this means studies can take many years, which may be unfavorable given the urgency of the current pandemic. The fewer number of animals studied also means that interpretation of data should be made with due caution. Additionally, researchers must make difficult choices about using primates for concept pathology studies or future vaccine tests. Finally, there are many ethical implications and legal constraints that limit research with nonhuman primates that may raise adverse public opinion. ${ }^{3}$ Nonhuman primate research is an indispensable tool for disease study, but like other animal testing, it must proceed with thoughtful consideration of various factors.

\section{Conclusion}

Animal models are vital in vaccine and therapeutics treatment development to identify mechanisms driving pathology. Nonhuman primates have been a critical model for respiratory infection to examine historically and at the same time as clinical trials with humans. Since they mimic human COVID-19 more faithfully than most other species because of anatomic, molecular, and physiological similarities, they have helped shape our current understanding of the COVID-19 pathophysiology tremendously. So far, established non-human primate models, mainly rhesus macaques, have recapitulated many aspects of human disease and are being used to test vaccine and other therapeutics efficacy and safety. Some species of nonhuman primates may potentially serve as more effective models of the disease, such as the African green monkey. Researchers are often constrained to using only a few animals, making the organization of data across multiple studies more essential. The unprecedented speed of COVID-19 research has been helped by the Internet's capacity for faster and more straightforward data sharing. Ultimately, the macaque models have assisted and will continue to benefit preventative methods and interventions for preclinical animal trials. These have paved the way for the ongoing human clinical trials. Further study and refinement of methodologies will enhance our knowledge as well as advance treatment and prevention of the ongoing pandemic.

\section{- Acknowledgments}

This work was initiated following the cancelation of my summer lab internship at the US Army Medical Research Institute of Infectious Diseases (USAMRIID) Division of Pathology. I thank Dr. Kevin Zeng for his mentorship and guidance throughout the process. The initial resources and publications he provided were central to my understanding of the topic. I am grateful for his encouragement during this research opportunity.

\section{- References}

1. Sohrabi, C.; Alsafi, Z.; O'Neill, N.; Khan, M.; Kerwan, A.; Al-Jabir, A.; Iosifidis, C.; Agha, R., World Health Organization declares global emergency: A review of the 2019 novel coronavirus (COVID-19). Int J Surg 2020, 76, 71-76.
2. Lythgoe, M. P.; Middleton, P., Ongoing Clinical Trials for the Management of the COVID-19 Pandemic. Trends Pharmacol Sci 2020, 41 (6), 363-382.

3. Miller, L. A.; Royer, C. M.; Pinkerton, K. E.; Schelegle, E. S., Nonhuman Primate Models of Respiratory Disease: Past, Present, and Future. ILAR J 2017, 58 (2), 269-280

4. Gretebeck, L. M.; Subbarao, K., Animal models for SARS and MERS coronaviruses. Curr Opin Virol 2015, 13, 123-9..

5. Shi, J.; Wen, Z.; Zhong, G.; Yang, H.; Wang, C.; Huang, B.; Liu, R.; He,X.; Shuai, L.; Sun, Z.; Zhao, Y.; Liu, P.; Liang, L.; Cui, P.; Wang, J.; Zhang, X.; Guan, Y.; Tan, W.; Wu, G.; Chen, H.; Bu, Z., Susceptibility of ferrets, cats, dogs, and other domesticated animals to SARS-coronavirus 2. Science 2020, 368 (6494), 1016-1020.

6. Kim, Y.I.; Kim, S. G.; Kim, S. M.; Kim, E. H.; Park, S.J.; Yu, K. M.; Chang, J. H.; Kim, E. J.; Lee, S.; Casel, M.A. B.; Um, J.; Song, M. S.; Jeong, H. W.; Lai, V. D.; Kim, Y.; Chin, B. S.; Park, J. S.; Chung, K. H.; Foo, S. S.; Poo, H.; Mo, I. P.; Lee, O. J.; Webby, R. J.; Jung, J. U.; Choi, Y. K., Infection and Rapid Transmission of SARS-CoV-2 in Ferrets. Cell Host Microbe 2020, 27 (5), 704-709 e2.

7. Bao, L.; Deng, W.; Huang, B.; Gao, H.; Liu,J.; Ren, L.; Wei, Q.; Yu, P.; Xu, Y.; Qi, F.; Qu, Y.; Li, F.; Lv, Q.; Wang, W.; Xue, J.; Gong, S.; Liu, M.; Wang, G.; Wang, S.; Song, Z.; Zhao, L.; Liu, P.; Zhao, L.; Ye, F.; Wang, H.; Zhou, W.; Zhu, N.; Zhen, W.; Yu, H.; Zhang, X.; Guo, L.; Chen, L.; Wang, C.; Wang, Y.; Wang, X.; Xiao, Y.; Sun, Q.; Liu, H.; Zhu, F.; Ma, C.; Yan, L.; Yang, M.; Han, J.; Xu, W.; Tan, W.; Peng, X.; Jin, Q.; Wu, G.; Qin, C., The pathogenicity of SARS-CoV-2 in hACE2 transgenic mice. Nature 2020, 583 (7818), 830-833.

8. Dinnon, K. H.; Leist, S. R.; Schafer, A.; Edwards, C. E.; Martinez, D. R.; Montgomery, S. A.; West, A.; Yount, B. L.; Hou, Y. J.; Adams, L. E.; Gully, K. L.; Brown, A. J.; Huang, E.; Bryant, M. D.; Choong, I. C.; Glenn, J. S.; Gralinski, L. E.; Sheahan, T. P.; Baric, R. S., A mouse-adapted SARS-CoV-2 model for the evaluation of COVID-19 medical countermeasures. bioRxiv 2020.

9. Golden, J. W.; Cline, C. R.; Zeng, X.; Garrison, A. R.; Carey, B. D.; Mucker, E. M.; White, L. E.; Shamblin, J. D.; Brocato, R. L.; Liu, J.; Babka, A. M.; Rauch, H. B.; Smith, J. M.; Hollidge, B. S.; Fitzpatrick, C.; Badger, C. V.; Hooper, J. W., Human angiotensinconverting enzyme 2 transgenic mice infected with SARS-CoV-2 develop severe and fatal respiratory disease. JCI Insight 2020, 5 (19). entomopathogenic Bacillus thuringiensis isolates on the midgut of the yellow mealworm larvae. Medycyna Wet 2007. 63(9):1049-51.

10. Smith, T. R. F.; Patel, A.; Ramos, S.; Elwood, D.; Zhu, X.; Yan, J.; Gary, E. N.; Walker, S. N.; Schultheis, K.; Purwar, M.; Xu, Z.; Walters, J.; Bhojnagarwala, P.; Yang, M.; Chokkalingam, N.; Pezzoli, P.; Parzych, E.; Reuschel, E. L.; Doan, A.; Tursi, N.; Vasquez, M.; Choi, J.; Tello-Ruiz, E.; Maricic, I.; Bah, M. A.; Wu, Y.; Amante, D.; Park, D. H.; Dia, Y.; Ali, A. R.; Zaidi, F. I.; Generotti, A.; Kim, K. Y.; Herring, T. A.; Reeder, S.; Andrade, V. M.; Buttigieg, K.; Zhao, G.; Wu, J. M.; Li, D.; Bao, L.; Liu, J.; Deng, W.; Qin, C.; Brown, A. S.; Khoshnejad, M.; Wang, N.; Chu, J.; Wrapp, D.; McLellan, J. S.; Muthumani, K.; Wang, B.; Carroll, M. W.; Kim, J. J.; Boyer, J.; Kulp, D. W.; Humeau, L.; Weiner, D. B.; Broderick, K. E., Immunogenicity of a DNA vaccine candidate for COVID-19. Nat Commun 2020, 11 (1), 2601.

11. Chan, J. F.; Zhang, A. J.; Yuan, S.; Poon, V. K.; Chan, C. C.; Lee, A. C.; Chan, W. M.; Fan, Z.; Tsoi, H. W.; Wen, L.; Liang, R.; Cao, J.; Chen, Y.; Tang, K.; Luo, C.; Cai, J.P.; Kok, K. H.; Chu, H.; Chan, K. H.; Sridhar, S.; Chen, Z.; Chen, H.; To, K. K.; Yuen, K. Y., Simulation of the clinical and pathological manifestations of Coronavirus Disease 2019 (COVID-19) in golden Syrian hamster 
model: implications for disease pathogenesis and transmissibility. Clin Infect Dis 2020.

12. Friedman, H.; Ator, N.; Haigwood, N.; Newsome, W.; Allan, J. S.; Golos, T. G.; Kordower, J. H.; Shade, R. E.; Goldberg, M. E.; Bailey, M. R.; Bianchi, P., The Critical Role of Nonhuman Primates in Medical Research. Pathog Immun 2017, 2 (3), 352-365.

13. Blanco-Melo, D.; Nilsson-Payant, B. E.; Liu, W. C.; Uhl, S.; Hoagland, D.; Moller, R.; Jordan, T. X.; Oishi, K.; Panis, M.; Sachs, D.; Wang, T. T.; Schwartz, R. E.; Lim, J. K.; Albrecht, R. A.; tenOever, B. R., Imbalanced Host Response to SARS-CoV-2 Drives Development of COVID-19. Cell 2020, 181 (5), 1036-1045 e9.

14. Leroy, E. M.; Ar Gouilh, M.; Brugere-Picoux, J., The risk of SARS-CoV-2 transmission to pets and other wild and domestic animals strongly mandates a one-health strategy to control the COVID-19 pandemic. One Health 2020, 10, 100133.

15. Rockx, B.; Kuiken, T.; Herfst, S.; Bestebroer, T.; Lamers, M. M.; Oude Munnink, B. B.; de Meulder, D.; van Amerongen, G.; van den Brand,J.; Okba, N. M. A.; Schipper, D.; van Run, P.; Leijten,L.; Sikkema, R.; Verschoor, E.; Verstrepen, B.; Bogers, W.; Langermans, J.; Drosten, C.; Fentener van Vlissingen, M.; Fouchier, R.; de Swart, R.; Koopmans, M.; Haagmans, B. L., Comparative pathogenesis of COVID-19, MERS, and SARS in a nonhuman primate model. Science 2020, 368 (6494), 1012-1015.

16. Munster, V. J.; Feldmann, F.; Williamson, B. N.; van Doremalen, N.; Perez-Perez, L.; Schulz, J.; Meade-White, K.; Okumura, A.; Callison,J.; Brumbaugh,B.; Avanzato, V.A.; Rosenke, R.; Hanley, P.W.; Saturday, G.; Scott,D.; Fischer,E.R.; de Wit,E., Respiratory disease in rhesus macaques inoculated with SARS-CoV-2. Nature 2020, 585 (7824), 268-272.

17. Yu, J.; Tostanoski, L. H.; Peter, L.; Mercado, N. B.; McMahan, K.; Mahrokhian, S.H.; Nkolola,J.P.; Liu,J.; Li,Z.; Chandrashekar, A.; Martinez, D. R.; Loos, C.; Atyeo, C.; Fischinger, S.; Burke, J. S.; Slein, M. D.; Chen, Y.; Zuiani, A.; Lelis, F. J. N.; Travers, M.; Habibi, S.; Pessaint, L.; Van Ry, A.; Blade, K.; Brown, R.; Cook, A.; Finneyfrock, B.; Dodson, A.; Teow, E.; Velasco, J.; Zahn, R.; Wegmann, F.; Bondzie, E. A.; Dagotto, G.; Gebre, M. S.; He, X.; Jacob-Dolan, C.; Kirilova, M.; Kordana, N.; Lin, Z.; Maxfield, L. F.; Nampanya, F.; Nityanandam, R.; Ventura,J. D.; Wan, H.; Cai, Y.; Chen, B.; Schmidt, A. G.; Wesemann, D. R.; Baric, R. S.; Alter, G.; Andersen, H.; Lewis, M. G.; Barouch, D. H., DNA vaccine protection against SARS-CoV-2 in rhesus macaques. Science 2020, 369 (6505), 806-811.

18. Lu, S.; Zhao, Y.; Yu, W.; Yang, Y.; Gao, J.; Wang, J.; Kuang, D.; Yang, M.; Yang, J.; Ma, C.; Xu,J.; Qian, X.; Li, H.; Zhao, S.; Li, J.; Wang, H.; Long, H.; Zhou,J.; Luo, F.; Ding, K.; Wu, D.; Zhang, Y.; Dong, Y.; Liu, Y.; Zheng, Y.; Lin, X.; Jiao, L.; Zheng, H.; Dai, Q.; Sun, Q.; Hu, Y.; Ke, C.; Liu, H.; Peng, X., Comparison of nonhuman primates identified the suitable model for COVID-19. Signal Transduct Target There 2020, 5 (1), 157.

19. Woolsey, C.; Borisevich, V.; Prasad, A. N.; Agans, K. N.; Deer, D. J.; Dobias, N. S.; Heymann, J. C.; Foster, S. L.; Levine, C. B.; Medina, L.; Melody, K.; Geisbert, J. B.; Fenton, K. A.; Geisbert, T. W.; Cross, R. W., Establishment of an African green monkey model for COVID-19. bioRxiv 2020.

20. Yu, P.; Qi, F.; Xu, Y.; Li, F.; Liu, P.; Liu, J.; Bao, L.; Deng, W.; Gao, H.; Xiang, Z.; Xiao, C.; Lv, Q.; Gong, S.; Liu,J.; Song, Z.; Qu, Y.; Xue, J.; Wei, Q.; Liu, M.; Wang, G.; Wang, S.; Yu, H.; Liu, X.; Huang, B.; Wang, W.; Zhao, L.; Wang, H.; Ye, F.; Zhou, W.; Zhen, W.; Han, J.; Wu, G.; Jin, Q.; Wang,J.; Tan, W.; Qin, C., Age-related rhesus macaque models of COVID-19.

Animal Model Exp Med 2020, 3 (1), 93-97.

21.Wu, J.T.; Leung, K.; Bushman, M.; Kishore, N.; Niehus, R.; de Salazar, P. M.; Cowling, B. J.; Lipsitch, M.; Leung, G. M., Estimating clinical severity of COVID-19 from the transmission dynamics in Wuhan, China. Nat Med 2020, 26 (4), 506-510.

22. Shan, C.; Yao, Y. F.; Yang, X. L.; Zhou, Y.W.; Gao, G.; Peng, Y.; Yang, L.; Hu, X.; Xiong,J.; Jiang, R. D.; Zhang, H.J.; Gao,X. X.; Peng, C.; Min,J.; Chen, Y.; Si,H.R.; Wu,J.; Zhou, P.; Wang, Y.Y.; Wei, H.P.; Pang, W.; Hu,Z.F.; Lv, L. B.; Zheng, Y.T.; Shi, Z. L.; Yuan, Z. M., Infection with novel coronavirus (SARS-

CoV-2) causes pneumonia in Rhesus macaques. Cell Res 2020, 30 (8), 670-677.

23. Van Doremalen, N.; Haddock, E.; Feldmann, F.; Meade-White, K.; Bushmaker, T.; Fischer, R. J.; Okumura, A.; Hanley, P. W.; Saturday, G.; Edwards, N.J.; Clark, M. H. A.; Lambe, T.; Gilbert, S. C.; Munster, V.J., A single dose of ChAdOx1 MERS provides protective immunity in rhesus macaques. Sci Adv 2020, 6 (24), eaba8399.

24. Gao, Q.; Bao, L.; Mao, H.; Wang, L.; Xu, K.; Yang, M.; Li, Y.; Zhu, L.; Wang, N.; Lv, Z.; Gao, H.; Ge, X.; Kan, B.; Hu, Y.; Liu, J.; Cai, F.; Jiang, D.; Yin, Y.; Qin, C.; Li, J.; Gong, X.; Lou, X.; Shi, W.; Wu, D.; Zhang, H.; Zhu, L.; Deng,W.; Li, Y.; Lu, J.; Li, C.; Wang, X.; Yin, W.; Zhang, Y.; Qin, C., Development of an inactivated vaccine candidate for SARS-CoV-2. Science 2020, 369 (6499), 77-81.

25. De Wit, E.; Feldmann, F.; Cronin, J.; Jordan, R.; Okumura, A.; Thomas, T.; Scott, D.; Cihlar, T.; Feldmann, H., Prophylactic and therapeutic remdesivir (GS-5734) treatment in the rhesus macaque model of MERS-CoV infection. Proc Natl Acad Sci U S A 2020, 117 (12), 6771-6776.

26. Williamson, B. N.; Feldmann, F.; Schwarz, B.; Meade-White, K.; Porter, D. P.; Schulz, J.; van Doremalen, N.; Leighton, I.; Yinda, C. K.; Perez-Perez, L.; Okumura, A.; Lovaglio,J.; Hanley, P.W.; Saturday, G.; Bosio, C.M.; Anzick, S.; Barbian, K.; Cihlar, T.; Martens, C.; Scott, D. P.; Munster, V.J.; de Wit, E., Clinical benefit of remdesivir in rhesus macaques infected with SARSCoV-2. Nature 2020, 585 (7824), 273-276.

27. Rosenke, K.; Jarvis, M.A.; Feldmann, F.; Schwarz, B.; Okumura, A.; Lovaglio, J.; Saturday, G.; Hanley, P.W.; Meade-White, K.; Williamson, B. N.; Hansen, F.; Perez-Perez, L.; Leventhal, S.; Tang-Huau, T.L.; Nason, M.; Callison, J.; Haddock, E.; Scott, D.; Sewell, G.; Bosio, C. M.; Hawman, D.; de Wit, E.; Feldmann, H., Hydroxychloroquine Proves Ineffective in Hamsters and Macaques Infected with SARS-CoV-2. bioRxiv 2020.

28. Maisonnasse, P.; Guedj, J.; Contreras, V.; Behillil, S.; Solas, C.; Marlin, R.; Naninck, T.; Pizzorno, A.; Lemaitre,J.; Goncalves, A.; Kahlaoui, N.; Terrier, O.; Fang, R.H.T.; Enouf,V.; DereuddreBosquet, N.; Brisebarre, A.; Touret, F.; Chapon, C.; Hoen, B.; Lina, B.; Calatrava, M. R.; van der Werf, S.; de Lamballerie, X.; Le Grand, R., Hydroxychloroquine use against SARS-CoV-2 infection in non-human primates. Nature 2020, 585 (7826), 584-587.

\section{Authors}

Maggie $\mathrm{Hu}$ is a current senior at Urbana High School in Ijamsville, Maryland. She has always been passionate about the sciences; these interests and current events inspired her to take on this research endeavor. In the future, she hopes to help others through a career in the medical field. 